\title{
Experiment Design for Identification of Nonlinear Gray-box Models with Applica- tion to Industrial Robots
}

Erik Wernholt, Johan Löfberg

Division of Automatic Control

E-mail: erikw@isy.liu.se, johanl@isy.liu.se

9th March 2007

Report no.: LiTH-ISY-R-2774

Submitted to CDC 2007

Address:

Department of Electrical Engineering

Linköpings universitet

SE-581 83 Linköping, Sweden

WWW: http://WwW. control.isy.liu.se

AUTOMATIC CONTROL

REGLERTEKNIK

LINKÖPINGS UNIVERSITET

Technical reports from the Automatic Control group in Linköping are available from http://www. control.isy.liu.se/publications. 


\begin{abstract}
Experiment design involving selection of optimal experiment positions for nonlinear gray-box models is studied. From the derived Fisher information matrix, a convex optimization problem is posed. By considering the dual problem, the experiment design is efficiently solved with linear complexity in the number of candidate positions, compared to cubic complexity for the primal problem. In the numerical illustration, using an industrial robot, the parameter covariance is reduced by a factor of six by using the 15 optimal positions compared to using the optimal single position in all experiments.
\end{abstract}

Keywords: Identification, Experiment design, Robotics, Nonlinear Graybox models, Convex optimization 


\title{
Experiment Design for Identification of Nonlinear Gray-box Models with Application to Industrial Robots
}

\author{
Erik Wernholt and Johan Löfberg
}

\begin{abstract}
Experiment design involving selection of optimal experiment positions for nonlinear gray-box models is studied. From the derived Fisher information matrix, a convex optimization problem is posed. By considering the dual problem, the experiment design is efficiently solved with linear complexity in the number of candidate positions, compared to cubic complexity for the primal problem. In the numerical illustration, using an industrial robot, the parameter covariance is reduced by a factor of six by using the 15 optimal positions compared to using the optimal single position in all experiments.
\end{abstract}

\section{INTRODUCTION}

Experiment design has been treated in the literature for almost half a century in both statistics and engineering areas, where some basic references are [1] and [2]. A recent survey can also be found in [3]. The experiment design problem aims at finding the optimal experiment conditions, $\chi$, contained in some set $\chi$, that minimizes a criterion related to the expected outcome of the experiment under certain constraints.

For parameter estimation, the parameter accuracy is a function of both the experimental conditions and the estimator. It is common, see [2], to assume that the estimator is efficient in the sense that the parameter covariance matrix achieves the Cramer-Rao lower bound (inverse of the Fisher information matrix), which is a lower limit on the covariance matrix that can be obtained with an unbiased estimator. The Fisher information matrix can be obtained without specifying an estimator. Usually, an assumption of normally distributed noise is made. According to [2, p. 127], this is not very restrictive since, asymptotically, the covariance matrix in many cases will be the same even for other noise distributions. This is, for example, the case for the prediction error method [4].

The classical approach has then been to minimize some scalar measure of the (asymptotic) covariance matrix $P_{\theta}\left(\chi, \theta_{0}\right)$ with constraints on the input and/or the output power. Some commonly used measures are A-optimality $\operatorname{Tr} P_{\theta}\left(\chi, \theta_{0}\right), \quad$ D-optimality $\operatorname{det} P_{\theta}\left(\chi, \theta_{0}\right), \quad$ E-optimality $\lambda_{\max }\left(P_{\theta}\left(\chi, \theta_{0}\right)\right)$, and L-optimality $\operatorname{Tr}\left(W P_{\theta}\left(\chi, \theta_{0}\right)\right)$. An inherent problem in most experiment design problems is that the covariance matrix depends on the true system parameters $\theta_{0}$. These parameters are, at least partly, unknown or uncertain, otherwise there would be no need for an experiment. This has been handled in different ways in the literature. One could either assume a good

E. Wernholt and J. Löfberg are with the Department of Electrical Engineering, Linköpings universitet, SE-58183 Linköping, Sweden \{erikw, johanl\} @isy.liu.se prior estimate of $\theta_{0}$ and hope for the best, or use some robust experiment design methods. Various strategies have been suggested, including sequential design (iterate between parameter estimation and experiment design), Bayesian design (minimize the expected value over the prior parameter distribution) and min-max design (minimize the worst case when the parameters are contained in a given set). For references, see [5].

In addition to the problem of knowing $\theta_{0}$, experiment design problems are, in their original form, often intractable due to non-convex and infinite-dimensional constraints, as well as the problem of finding a signal realization which has the desired spectral properties. However, due to great advances in the optimization community, there exist today many useful methods to reformulate the experiment design problems into tractable convex optimization problems. Here, [6] is a good example, where a framework for this reformulation is presented, based on finite-dimensional parameterizations of the signal spectrum. Constraints on these spectra can be included, as well as quality constraints and robustness constraints as long as they can be rewritten as convex functions of the inverse (asymptotic) covariance matrix.

\section{PROBLEM DESCRIPTION}

The work in this paper is inspired by the problem of identification of flexibility parameters in a nonlinear industrial robot model [7]. The industrial robot is challenging for system identification since it is a multivariable, nonlinear, unstable, and highly resonant system. Consider therefore the nonlinear gray-box model as

$$
\begin{aligned}
& \dot{x}(t)=f(x(t), u(t), \theta), \\
& y(t)=h(x(t), u(t), \theta),
\end{aligned}
$$

with state vector $x(t) \in \mathbb{R}^{n_{x}}$, input $u(t) \in \mathbb{R}^{n_{u}}$, output $y(t) \in \mathbb{R}^{n_{y}}$, and the nonlinear functions $f(\cdot)$ and $h(\cdot)$, parameterized by $\theta \in \mathbb{R}^{d}$. A discrete-time feedback controller $^{1} u(t)=F(q)(r(t)-y(t))$ is used to stabilize the system, where $F(q)$ is the controller and $q$ is the difference operator. The experiment design problem involves finding the excitation signal $r(t)$ (and possibly $F(q)$ if that is a design variable) that minimizes the parameter uncertainty, given certain constraints on $r(t), u(t), x(t)$. This is a nonlinear optimal control problem which is extremely hard to solve. One option would be to apply a prediction error method for

\footnotetext{
${ }^{1}$ Physical modeling makes continuous-time gray-box models most natural. Still, a discrete-time controller will be used which gives that the excitation signal $r(t)$ in the experiment design is a discrete-time signal. For simplicity, blocks for sample and hold are left out in the sequel.
} 
the parameter estimation. A covariance matrix could then be calculated from the cost function. However, minimizing some measure of this covariance matrix would probably be a hard non-convex optimization problem. In addition comes the problem of obtaining a stable predictor for the nonlinear system.

In this paper, a different approach will be used, where we assume that $r(t)$ is a small perturbation around a certain operating point $\left(x_{0}, u_{0}\right)$, called position in this paper. The perturbation is assumed to be small enough to justify linearizion of the nonlinear system (1) and the use of linear theory. The identification will be carried out in the frequency domain by comparing the experimental FRF (Frequency Response Function) with the FRF obtained by linearizing the nonlinear model.

The information about the unknown parameters will differ between different positions. Therefore, given a limited total measurement time, one should perform experiments in the position(s) that contribute the most to the information about the unknown parameters. Here, a set of $Q$ candidate positions will be assumed and a total of $M$ experiments should be performed. For each position, a separate experiment design problem can be solved, as in [6], where the excitation signal is optimized in order to meet constraints and minimize the parameter uncertainty, given a fixed measurement time.

This will be a suboptimal solution to the overall experiment design problem since the optimization is done in two steps. However, in principle it is possible to solve the overall problem by parameterizing the excitation signal(s) $\left(n_{u} n_{f}\right.$ parameters), as in [6], for each position and then solve the resulting problem with $Q n_{f} n_{u}$ variables and additional constraints for each position. The optimization problem is still convex if the constraints are convex functions of the variables that define the excitation signals (for example, a power constraint). This is an interesting extension of the work presented here and preliminary studies show promising results.

The paper is organized as follows: In Section III, the Fisher information matrix is derived, assuming certain properties of the estimated FRFs. This enables the formulation of the experiment design problem in Section IV. The solution of this problem is discussed in Section $\mathrm{V}$ and a numerical illustration, using the industrial robot, is given in Section VI. Finally, some conclusions are made in Section VII.

\section{THE INFORMATION MATRIX}

In this section, we assume that information about the unknown parameters can be obtained by measuring the nonlinear system in $Q$ different positions. The complexvalued FRF measurements are assumed to have the following properties.

Assumption 1: Let $\hat{\mathcal{G}}^{(i)}(l) \in \mathbb{C}^{n}$ denote measurement $l$ in position $i$. For each position $i=1, \ldots, Q, m_{i}$ experiments will be carried out, giving a total of $M=\sum_{i=1}^{Q} m_{i}$ experiments. The measurements $\hat{\mathcal{G}}^{(i)}(l)$ can be written as

$$
\hat{\mathcal{G}}^{(i)}(l)=\mathcal{G}^{(i)}\left(\theta_{0}\right)+\eta^{(i)}(l),
$$

where $\mathcal{G}^{(i)}(\theta)$ is the model FRF for position $i$ and $\theta_{0}$ is the same for all positions. The measurement noise $\eta^{(i)}(l)$ is a zero mean, circular complex normally distributed random variable, independent (over $i$ and $l$ ) and identically distributed (over $l$ ) with covariance matrix $\Lambda_{0}^{(i)}$.

For FRF measurements with $n_{f}$ frequencies, $n$ would be $n=n_{u} n_{y} n_{f}$, see (13). Given the measurements and a model, one must come up with an estimator $\hat{\theta}_{M}$ for the unknown parameters. If the estimator is unbiased $\left(\mathrm{E} \hat{\theta}_{M}=\theta_{0}\right)$, the covariance of any such estimator is bounded by the Fisher information matrix $H$,

$$
\mathrm{E}\left[\left(\hat{\theta}_{M}-\theta_{0}\right)\left(\hat{\theta}_{M}-\theta_{0}\right)^{T}\right] \succeq H^{-1} .
$$

This bound is often referred to as the Cramer-Rao lower bound (CRLB). Next, the Fisher information matrix, $H$, as well as the maximum likelihood estimator are derived, given Assumption 1.

Theorem 1: Given Assumption 1, the Fisher information matrix is given by

$$
H=\sum_{i=1}^{Q} m_{i} H_{i}
$$

where $H_{i}$ is the information matrix in position $i$,

$$
H_{i}=2 \Re\left\{\overline{\Psi^{(i)}\left(\theta_{0}\right)}\left[\Lambda_{0}^{(i)}\right]^{-1}\left[\Psi^{(i)}\left(\theta_{0}\right)\right]^{T}\right\},
$$

with $\Psi^{(i)}(\theta)=\frac{d}{d \theta} \mathcal{G}^{(i)}(\theta) \in \mathbb{C}^{d \times n}$ and $\overline{(\cdot)}$ denoting complex conjugate.

Proof: The information matrix $H$ is calculated as

$$
H=\left.\mathrm{E}\left[\frac{d}{d \theta} \log f(\hat{\mathcal{G}} \mid \theta)\right]\left[\frac{d}{d \theta} \log f(\hat{\mathcal{G}} \mid \theta)\right]^{T}\right|_{\theta=\theta_{0}},
$$

where $f(\hat{\mathcal{G}} \mid \theta)$ is the conditional probability density function (PDF) for $\hat{\mathcal{G}}$, given $\theta$, where $\hat{\mathcal{G}}$ denotes the set of measurements $\left\{\hat{\mathcal{G}}^{(i)}(l), l=1, \ldots, m_{i}, i=1, \ldots, Q\right\}$. Independent measurements gives that

$$
f(\hat{\mathcal{G}} \mid \theta)=\prod_{i=1}^{Q} \prod_{l=1}^{m_{i}} f\left(\hat{\mathcal{G}}^{(i)}(l) \mid \theta\right)=\prod_{i=1}^{Q} \prod_{l=1}^{m_{i}} f_{\eta^{(i)}}\left(\mathcal{E}^{(i)}(l, \theta)\right)
$$

where $\mathcal{E}^{(i)}(l, \theta)=\hat{\mathcal{G}}^{(i)}(l)-\mathcal{G}^{(i)}(\theta)$ and $f_{\eta^{(i)}}(x)$ is the PDF for the noise $\eta^{(i)}(l) \in \mathbb{C}^{n}$. Zero mean circular complex normally distributed noise has the PDF [8, p. 437]

$$
f_{\eta^{(i)}}(x)=\frac{1}{\pi^{n} \operatorname{det}\left(\Lambda_{0}^{(i)}\right)} \exp \left(-x^{H}\left(\Lambda_{0}^{(i)}\right)^{-1} x\right),
$$

which gives

$$
\begin{aligned}
\log f(\hat{\mathcal{G}} \mid \theta)=-\sum_{i=1}^{Q} & \sum_{l=1}^{m_{i}}\left(n \log \pi+\log \operatorname{det} \Lambda_{o}^{(i)}+\right. \\
& {\left.\left[\mathcal{E}^{(i)}(l, \theta)\right]^{H}\left[\Lambda_{o}^{(i)}\right]^{-1} \mathcal{E}^{(i)}(l, \theta)\right), }
\end{aligned}
$$

and

$$
\left.\frac{d}{d \theta} \log f(\hat{\mathcal{G}} \mid \theta)\right|_{\theta=\theta_{0}}=2 \Re\left\{\overline{\Psi^{(i)}\left(\theta_{0}\right)}\left[\Lambda_{0}^{(i)}\right]^{-1} \eta^{(i)}(l)\right\} .
$$


Since $\eta^{(i)}(l)$ is independent over $i$ and $l$, the information matrix $H$ is finally given by

$$
\begin{aligned}
H & =\sum_{i=1}^{Q} \sum_{l=1}^{m_{i}} 4 \operatorname{Cov} \Re\left\{\overline{\Psi^{(i)}\left(\theta_{0}\right)}\left[\Lambda_{0}^{(i)}\right]^{-1} \eta^{(i)}(l)\right\} \\
& =\sum_{i=1}^{Q} \sum_{l=1}^{m_{i}} 2 \Re\left\{\operatorname{Cov} \overline{\Psi^{(i)}\left(\theta_{0}\right)}\left[\Lambda_{0}^{(i)}\right]^{-1} \eta^{(i)}(l)\right\} \\
& =\sum_{i=1}^{Q} m_{i} \cdot 2 \Re\left\{\overline{\Psi^{(i)}\left(\theta_{0}\right)}\left[\Lambda_{0}^{(i)}\right]^{-1}\left[\Psi^{(i)}\left(\theta_{0}\right)\right]^{T}\right\},
\end{aligned}
$$

where the fact that $\eta^{(i)}(l)$ is a circular complex random vector has been used. [If $x$ is a zero mean circular complex random vector $\left(\operatorname{Cov} x=\mathrm{E} x x^{H}, \mathrm{E}\left\{x x^{T}\right\}=0\right.$ ), then $y=A x$, with $A$ a complex matrix, is circular complex as well. For a circular complex random vector, $\operatorname{Cov} \Re\{x\}=$ $0.5 \Re\{\operatorname{Cov} x\}$ is also easy to verify.]

Corollary 1: Given Assumption 1, the maximum likelihood estimator $\hat{\theta}_{M}^{M L}=\arg \max _{\theta} \log f(\hat{\mathcal{G}} \mid \theta)$ is given by

$$
\hat{\theta}_{M}^{M L}=\arg \min _{\theta} \sum_{i=1}^{Q} \sum_{l=1}^{m_{i}}\left[\mathcal{E}^{(i)}(l, \theta)\right]^{H}\left[\Lambda_{o}^{(i)}\right]^{-1} \mathcal{E}^{(i)}(l, \theta)
$$

, with $\mathcal{E}^{(i)}(l, \theta)=\hat{\mathcal{G}}^{(i)}(l)-\mathcal{G}^{(i)}(\theta)$. The estimator is also efficient, in the sense that the Cramer-Rao lower bound in Theorem 1 is attained asymptotically $(M \rightarrow \infty)$.

Proof: The likelihood function is given by (6) which immediately gives (7). Efficiency follows from classical results for the maximum likelihood estimator in case of independent measurements (see, for example, [4, p. 215]).

\section{The EXPERIMENT DESIGN PROBlem}

Consider the information matrix $H$ in Theorem 1. The experiment design problem can be stated as follows: Given $Q$ different candidate positions and a total number of $M$ experiments to be carried out, choose the numbers $m_{i}, i=$ $1, \ldots, Q$ to minimize some measure of the CRLB $H^{-1}$.

Here, the D-optimal design problem will be considered, which can be posed as

$$
\begin{array}{ll}
\text { minimize } & \log \operatorname{det}\left[\frac{1}{M} \sum_{i=1}^{Q} m_{i} H_{i}\right]^{-1}, \\
\text { subject to } & m \succeq 0, \quad \mathbf{1}^{T} m=M, \quad m \in \mathbb{Z}^{Q},
\end{array}
$$

where $H_{i}=H_{i}^{T} \in \mathbb{R}^{d \times d}$ is the information matrix from position $i$, see (5). The factor $1 / M$ is added to obtain the average information matrix. This is a combinatorial experiment design problem since the $m_{i}$ are constrained to be integers. Given a large number of candidates $Q$, the problem will relatively quickly become intractable ${ }^{2}$. This problem fits into the framework of [10, Chapter 7.5] where also E-optimal and A-optimal designs are treated. See also [11, Section 2.4]. When $M$ is large, a reasonable approximate solution can

\footnotetext{
${ }^{2}$ The mixed integer conic solver in YALMIP [9] is capable of solving (8) for the design problem presented in Section VI for a few hundred positions.
}

be obtained by considering the relaxed experiment design problem

$$
\begin{aligned}
& \operatorname{minimize} \quad \log \operatorname{det}\left[\sum_{i=1}^{Q} \lambda_{i} H_{i}\right]^{-1}, \\
& \text { subject to } \lambda \succeq 0, \quad \mathbf{1}^{T} \lambda=1,
\end{aligned}
$$

For the relaxed problem, $M$ will no longer affect the solution $\lambda^{*}$ and the CRLB $H^{-1}$ is thus inversely proportional to the number of experiments, $M$, that will be carried out. For the combinatorial problem (8), this is only asymptotically true (cf. Figure 4).

$>$ From the solution $\lambda^{*}$ of the relaxed problem (9) it is possible to obtain a suboptimal solution $\tilde{m}$ to the combinatorial problem (8) as ${ }^{3} \tilde{m}=\operatorname{round}\left(M \lambda^{*}\right)$. Even though this is a suboptimal solution, it is possible to evaluate the solution by comparing the cost $V\left(M \lambda^{*}\right)$ and $V(\tilde{m})$, where $V(x)=\left[\log \operatorname{det} \sum_{i} x_{i} H_{i}\right]^{-1} \cdot V\left(M \lambda^{*}\right)$ is a lower bound on $V\left(m^{*}\right)$, so we have $V\left(M \lambda^{*}\right) \leq V\left(m^{*}\right) \leq V(\tilde{m})$. If the difference $V(\tilde{m})-V\left(M \lambda^{*}\right)$ is small, then $V(\tilde{m})$ must be close to $V\left(m^{*}\right)$. Note that when $M$ is small, the difference $V(\tilde{m})-V\left(M \lambda^{*}\right)$ will be large, but that is usually the case also for $V\left(m^{*}\right)-V\left(M \lambda^{*}\right)$. (This is, for example, evident for $M=1$ in the numerical illustration, see Figure 4.)

\section{Solving the ExPeriment Design Problem}

Determinant maximization problems arise in many fields (see [11] for an overview) and efficient solvers for this class of optimization problems are available. In fact, any semidefinite programming solver can be used to solve determinant maximization problems, although it requires cumbersome reformulations [12]. A recent example of a specialized solver is SDPT3 [13], which is designed to solve conic programming problems (which includes linear, quadratic, and semidefinite programs) with additional logarithmic barrier terms in the objective. This includes the determinant maximization problem (9) as a special case.

The purpose of this section is to motivate an alternative representation of (9), derived from duality. A common misconception is that when a so called primal-dual solver such as SDPT3 is used, it does not make any difference how the model is implemented, in terms of primal and dual models. However, this is a dangerous fallacy.

Although the details are beyond the scope of this paper, we note that the complexity of solving the determinant optimization problem (9), with $d \ll Q$, can be shown to be at least cubic in $Q$ (a linear system of size $Q \times Q$ has to be solved repeatedly during the solution process), when the model is interpreted in a dual sense $e^{4}$. Accordingly, the

\footnotetext{
${ }^{3}$ In addition, the following is done to fulfill $\mathbf{1}^{T} m=M$ while $\operatorname{sum}(\mathrm{m}) \sim=\mathrm{M}$

if $\operatorname{sum}(\mathrm{m})>\mathrm{M},[\operatorname{dum}, \mathrm{k}]=\max (\mathrm{m}-\mathrm{l} \mathrm{ambda} * \mathrm{M}) ; \mathrm{m}(\mathrm{k})=\mathrm{m}(\mathrm{k})-1$;

else $[$ dum, $\mathrm{k}]=\min (\mathrm{m}-\mathrm{lambda} * \mathrm{M}) ; \mathrm{m}(\mathrm{k})=\mathrm{m}(\mathrm{k})+1$; end end

${ }^{4}$ Following the primal-dual definitions in [13], which also is the way the modeling language YALMIP [9] and most people in the control community interprets problems.
} 


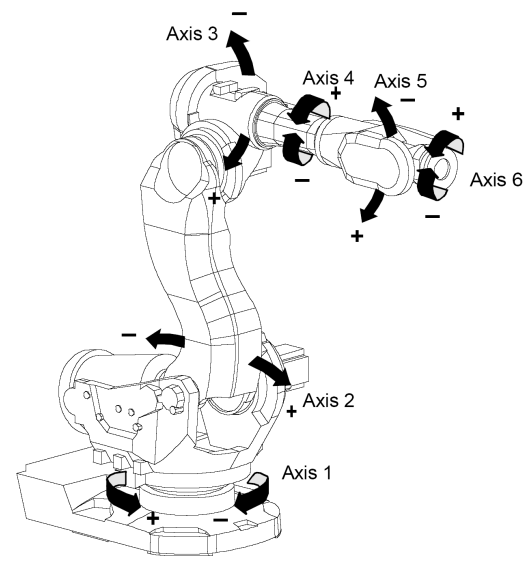

Fig. 1. The ABB manipulator IRB 6600 .

complexity of solving (9) scales badly with an increasing number of positions, $Q$.

Hence, blindly stating and solving the problem (9) can easily lead to unnecessarily complex models. Instead, we derive the dual of (9) as (cf. (3.4) in [11])

$$
\begin{aligned}
\operatorname{minimize} & \log \operatorname{det} W^{-1}, \\
\text { subject to } & W=W^{T} \succ 0, \\
& \operatorname{Tr}\left(W H_{i}\right) \leq d, \quad i=1, \ldots, Q .
\end{aligned}
$$

This problem, once again interpreted in a dual sense, has only $d(d+1) / 2$ variables (the variables parameterizing the symmetric matrix $W$ ). An increasing number of positions now instead leads to a growing number of linear inequality constraints. Although the complexity of solving a semidefinite program depends on the number of linear inequalities in the dual, it scales much better than increasing the number of variables. Roughly speaking, while complexity with respect to the number of variables in the dual for a standard SDP solver is at least cubic, the computational complexity with respect to the number of linear inequality constraints is essentially linear.

Note that the original variables $\lambda$ are dual variables related to the inequality constraints in the model (10). Hence, after solving the problem using the primal-dual solver SDPT3, the original variables are easily recovered.

\section{NUMERICAL ILLUSTRATION}

As a numerical illustration, we will use an industrial robot, see Figure 1. First, the robot model will be described in Section VI-A. The measurements $\hat{\mathcal{G}}^{(i)}(l)$ will, for each experiment, be the FRF estimate for a number number of frequencies, which is explained in Section VI-B. In Section VI-C, the experiment design problem will be solved using YALMIP [9] and SDPT3 [13] and the results are presented in Section VI-D.

\section{A. Robot model}

The model comes from [7] and consists of a kinematic chain of rigid bodies representing the manipulator arms. The arms are linked together in joints by gearboxes and motors, and due to flexibilities in the gearboxes, spring-damper pairs are introduced between all motors and arms. Two additional spring-damper pairs are introduced in some joints to model bearing flexibilities. The model dynamics can be described by the following set of equations.

$$
\begin{gathered}
{\left[\begin{array}{c}
\dot{v}_{b} \\
\dot{v}_{a}
\end{array}\right]=M_{a}^{-1}\left(q_{b}, q_{a}\right)\left[c\left(q_{b}, q_{a}, v_{b}, v_{a}\right)+g\left(q_{b}, q_{a}\right)-\left[\begin{array}{c}
\tau_{b} \\
\tau_{g}
\end{array}\right]\right],} \\
\dot{v}_{m}=M_{m}^{-1}\left(\tau_{g}+u\right), \quad \dot{q}_{a}=v_{a}, \quad \dot{q}_{m}=v_{m}, \quad \dot{q}_{b}=v_{b}, \\
\tau_{g}=K_{g}\left(q_{a}-q_{m}\right)+C_{g}\left(v_{a}-v_{m}\right), \quad \tau_{b}=K_{b} q_{b}+C_{b} v_{b},
\end{gathered}
$$

where $q_{a}, q_{m}, q_{b}$ are angular positions for arms, motors, and bearings, respectively. The matrix $M_{a}\left(q_{b}, q_{a}\right)$ is the inertia matrix for the arms and $M_{m}$ is the diagonal inertia matrix for the motors. The Coriolis and centrifugal torques are described by the function $c\left(q_{b}, q_{a}, v_{b}, v_{a}\right)$, and $g\left(q_{b}, q_{a}\right)$ represents gravity torque. The time $t$ is omitted in the expressions. The matrices $K_{g}, C_{g}$ and $K_{b}, C_{b}$ describes the flexibilities and are here diagonal.

The model used in the numerical illustration has threedimensional spring-damper pairs in the three main axis joints (1-3) and one-dimensional spring-damper pairs (gearbox) in the joints (4-6), see Figure 1. This gives 12 spring-damper pairs and 18 degrees of freedom $(6$ arms +6 motors $+2 \cdot 3$ joints having bearing flexibilities) and the nonlinear gray-box model (1) will have 36 states. As output, the motor angular velocity will be used, $y=v_{m}$. The rigid body parameters are known from a CAD (Computer Aided Design) model and the nominal spring-damper parameters are obtained from the identification described in [7]. For the experiment design, $\theta$ will contain the 12 spring parameters $(d=12)$. In addition, these are scaled by the nominal values such that $\theta_{0}=\mathbf{1}$.

The model equations are linearized in $Q$ different positions $q_{m}=q_{m}^{(i)}, i=1, \ldots, Q$, with $v_{m}=v_{a}=v_{b}=0$, $u=\tau_{g}$ and $q_{b}, q_{a}$ the solution to $g\left(q_{b}, q_{a}\right)=\left[\begin{array}{cc}\tau_{b}^{T} & \tau_{g}^{T}\end{array}\right]^{T}$. (For simplicity, $q_{a}=q_{m}, q_{b}=0$ can be used with minor errors if the springs are reasonably stiff.) From a resulting continuous-time transfer function $G_{c}^{(i)}(s, \theta)$, the discretetime FRF $G^{(i)}\left(e^{j \omega T_{s}}, \theta\right)$ is given by [14, Chapter 4.5]

$$
G^{(i)}\left(e^{j \omega T_{s}}, \theta\right)=\frac{1-e^{-j \omega T_{s}}}{j \omega T_{s}} G_{c}^{(i)}(j \omega, \theta),
$$

where higher-order terms in the Poisson summation formula have been neglected. These terms are small since $T_{s}$ is small and frequencies above $\pi / T_{s} \mathrm{rad} / \mathrm{s}$ are well attenuated by the dynamics in the torque controllers (left out in the modeling).

\section{B. Measurements}

Assume a closed-loop setup, $y(t)=G(q) u(t)+v(t)$, $u(t)=F(q)(r(t)-y(t))$, where $y, r \in \mathbb{R}^{n_{y}}$ and $u \in$ $\mathbb{R}^{n_{u}}$. The FRF estimate is then calculated from the DFT matrices $\mathbf{Y}\left(\omega_{k}\right) \in \mathbb{C}^{n_{y} \times n_{u}}$ and $\mathbf{U}\left(\omega_{k}\right) \in \mathbb{C}^{n_{u} \times n_{u}}$ like $\hat{G}\left(e^{j \omega_{k} T_{s}}\right)=\mathbf{Y}\left(\omega_{k}\right) \mathbf{U}^{-1}\left(\omega_{k}\right)$, where each column of $\mathbf{Y}\left(\omega_{k}\right)$ and $\mathbf{U}\left(\omega_{k}\right)$ contain the DFT of the sampled data from each sub-experiment (for a multivariable system, $n_{u}$ subexperiments are needed to form the FRF estimate). As excitation, an orthogonal multisine signal will be used, which 


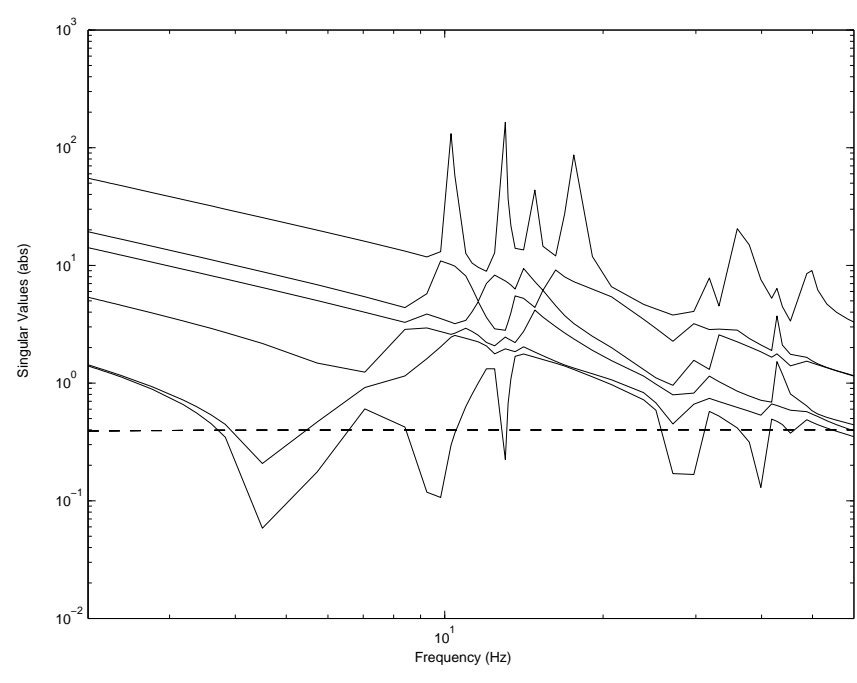

Fig. 2. Singular values for the model (11) in position $q_{m}=0$ together with the inverse controller $F^{-1}$ (dashed line).

gives $\mathbf{R}\left(\omega_{k}\right)=R_{0}\left(\omega_{k}\right) T$, where $R_{0}\left(\omega_{k}\right)$ is a scalar and $T$ is an orthogonal permutation matrix with $T T^{H}=n_{y} I$ (if $n_{y}=n_{u}$ ). Assuming $v(t)$ being noise, independent and identically distributed for different sub-experiments, with power spectrum $\Phi_{v}\left(\omega_{k}\right) \in \mathbb{C}^{n_{y} \times n_{y}}$ gives the uncertainty $\Lambda_{\hat{G}_{v}}^{(i)}\left(\omega_{k}\right)$ in the FRF estimate $\hat{G}_{v}^{(i)}\left(e^{j \omega_{k} T_{s}}\right)$ as follows (omitting the frequency argument).

$$
\Lambda_{\hat{G}_{v}}^{(i)}=\frac{n_{y}}{\Phi_{r_{0}}}\left[\left(G^{(i)}+F^{-1}\right)^{H}\left(G^{(i)}+F^{-1}\right)\right]^{T} \otimes \Phi_{v},
$$

where $\Phi_{r_{0}}\left(\omega_{k}\right)=\left|R_{0}\left(\omega_{k}\right)\right|^{2}$. It should be mentioned that (12) is an approximation since the FRF will be slightly biased due to data collection under feedback control. For details and proof, see [15].

In the numerical illustration, $\Phi_{v}(\omega)=C_{v} \omega^{2} I$ and $\Phi_{r_{0}}\left(\omega_{k}\right)=C_{r}$ for $n_{f}=50$ logarithmically spaced frequencies between 1 and $60 \mathrm{~Hz}$ and zero otherwise. The uncertainty will therefore be scaled by the factor $C_{v} / C_{r}$. A diagonal PI controller is used and the singular values for $F^{-1}\left(e^{j \omega_{k} T_{s}}\right)$ as well as $G^{(i)}\left(e^{j \omega_{k} T_{s}}, \theta_{0}\right)$ for position $q_{m}=0$ can be seen in Figure 2. To view all 36 elements, see [7].

For the experiment design, $\Psi^{(i)}(\theta)=\frac{d}{d \theta} \mathcal{G}^{(i)}(\theta)$ and $\Lambda_{0}^{(i)}$ are needed to calculate the information matrix $H_{i}$ in (5). Here, the model $\mathcal{G}^{(i)}(\theta)$ is given by

$$
\mathcal{G}^{(i)}(\theta)=\left[\begin{array}{c}
G_{v}^{(i)}\left(e^{j \omega_{1} T_{s}}, \theta\right) \\
\vdots \\
G_{v}^{(i)}\left(e^{j \omega_{n} T_{s}}, \theta\right)
\end{array}\right],
$$

where $G_{v}^{(i)}\left(e^{j \omega_{k} T_{s}}, \theta\right)$ is the model FRF in (11). The gradient $\Psi^{(i)}(\theta)$ is calculated numerically by central differences. The covariance matrix $\Lambda_{0}^{(i)}$ is a block-diagonal matrix with

\footnotetext{
${ }^{5}$ To simplify the notation, $G_{v}=\operatorname{vec}(G)$ will be used for the vectorized version of $G$, where the columns are stacked on top of each other.
}

$\Lambda_{\hat{G}_{v}}^{(i)}\left(\omega_{1}\right), \ldots, \Lambda_{\hat{G}_{j}}^{(i)}\left(\omega_{n_{f}}\right)$ on the diagonal. As candidate positions, all possible combinations of

$$
\begin{aligned}
& q_{m 1}=\{0,45,90\}, \quad q_{m 2}=\{-60,-45, \cdots, 90\}, \\
& q_{m 3}=\{-165,-150, \cdots, 60\}, \quad q_{m 4}=\{0,45,90\}, \\
& q_{m 5}=\{-90,-45,0,45,90\}, \quad q_{m 6}=0,
\end{aligned}
$$

will be used, giving a total of $Q=7920$ candidate positions. $q_{m 6}=0$ is used since the payload is symmetric with respect to $q_{m 6}$. The position in Figure 1 corresponds to $q_{m}=0$.

\section{Solution using YALMIP and SDPT3}

The experiment design problem (9) (or actually the dual problem (10)) will now be solved using YALMIP [9] and SDPT3 [13]. Although YALMIP by default interprets problems in a dual setting, it is possible to use the automatic dualization feature. If this is used, the dual problem (10) is automatically derived from (9) and solved, conveniently allowing us to solve the most efficient model without manually deriving any duals. This is particularly useful if we want to change the original problem (9) by adding additional constraints or terms in the objective, without deriving a new dual problem by hand. The original $\lambda$ variables are automatically recovered by YALMIP from dual variables in SDPT3.

As we mentioned in Section V, the computational complexity of solving the design problem is roughly linear when dualized, and cubic when interpreted in the naive original form. Although a dualized model gives us several orders of magnitudes faster computations, there is still room for improvement. The solver SDPT3 is developed with large sparse problems in mind, whereas our problem turns out to be dense. By changing one line of linear algebra code in SDPT3 from sparse to dense, the solver ran approximately four times faster on the dualized model. The optimization models were evaluated experimentally ${ }^{6}$ and the computation times are illustrated in Figure 3. Note that the primal model only could be solved up to $Q \approx 4000$ due to memory limitations. The curves confirm the linear growth in $Q$ for the dualized models, whereas the naive primal formulation grows slower than the expected cubic, but still requires far more computational effort. This simply indicates that there are other major contributors to the computations than the solution of the linear system, which was used for the crude asymptotic complexity estimate.

\section{Results}

The solution to the relaxed experiment design problem (9) with $Q=7920$ candidate positions gives a $\lambda^{*}$ with 15 nonzero elements. The corresponding positions and $\lambda^{*}$ values are presented in Table I and Figure 5 together with the geometric mean $\left(\operatorname{det} H_{i}^{-1}\right)^{1 / d}$ as a measure of the covariance matrix. In addition are the positions presented with min, max, and median covariance. These values should be compared

\footnotetext{
${ }^{6}$ Using a $1.7 \mathrm{GHz}$ Pentium M processor with $1 \mathrm{~GB}$ RAM. A complete implementation, with illustrative data, can be found at http://control.ee.ethz.ch/ joloef/wiki/pmwiki.php?n=Examples.Experiment
} 


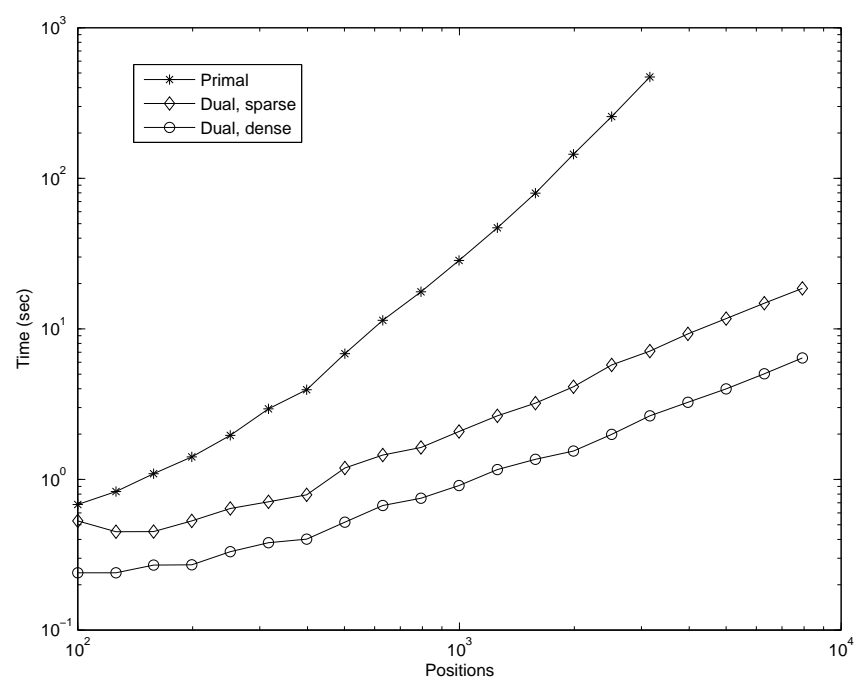

Fig. 3. Computation time when solving the primal and dual problems as a function of the number of candidate positions $Q$.

\section{TABLE I}

THE SOLUTION TO THE RELAXED EXPERIMENT DESIGN PROBLEM (9). THE COVARIANCE IS THE GEOMETRIC MEAN $\left(\operatorname{det} H_{i}^{-1}\right)^{1 / d}$. IN ADDITION ARE THE POSITIONS PRESENTED WITH MIN, MAX, AND MEDIAN COVARIANCE.

\begin{tabular}{r|rrrrr|r|l}
\hline Pos & $q_{m 1}$ & $q_{m 2}$ & $q_{m 3}$ & $q_{m 4}$ & $q_{m 5}$ & Cov & $\lambda$ \\
\hline 1 & 0 & -30 & -75 & 0 & 0 & 15.4 & 0.1083 \\
2 & 45 & -60 & -30 & 0 & 45 & 19.6 & 0.0256 \\
3 & 45 & -60 & -30 & 45 & 45 & 21.2 & 0.0093 \\
4 & 45 & -30 & -30 & 45 & -45 & 6.3 & 0.1606 \\
5 & 45 & -30 & 60 & 45 & 90 & 7.4 & 0.0509 \\
6 & 45 & 15 & -150 & 90 & 90 & 20.1 & 0.0350 \\
7 & 45 & 15 & -15 & 90 & -45 & 26.6 & 0.0881 \\
8 & 45 & 45 & -150 & 45 & -90 & 15.4 & 0.0895 \\
9 & 45 & 45 & -105 & 0 & -90 & 26.4 & 0.0927 \\
10 & 90 & -60 & -60 & 90 & 90 & 23.9 & 0.0451 \\
11 & 90 & -30 & -30 & 90 & 90 & 12.9 & 0.0238 \\
12 & 90 & -30 & 0 & 90 & -45 & 40.6 & 0.0225 \\
13 & 90 & 0 & -105 & 45 & -90 & 23.9 & 0.0192 \\
14 & 90 & 30 & 30 & 0 & 90 & 11.7 & 0.1300 \\
15 & 90 & 60 & 15 & 0 & 0 & 24.7 & 0.0993 \\
\hline Min & 45 & 15 & -135 & 45 & 45 & 5.9 & \\
Med & 45 & 30 & -90 & 90 & -90 & 122.6 & \\
Max & 0 & 60 & -90 & 90 & 0 & 673.6 & \\
\hline
\end{tabular}

with a covariance value of 1 for the optimal solution ${ }^{7}$ to the relaxed problem.

As was mentioned in Section IV, a sub-optimal solution to the original combinatorial experiment design problem can be obtained as $\tilde{m}=\operatorname{round}\left(M \lambda^{*}\right)$. The resulting covariance, $V(\tilde{m})$ with $V(x)=\left(\operatorname{det} \sum_{i} x_{i} H_{i}\right)^{-1 / d}$, is plotted in Figure 4 together with the relaxed value $V\left(M \lambda^{*}\right)$ as a function of $M$. In addition are the corresponding values plotted for the optimal single position (min in Table I) and the mean value using $M$ random positions.

The difference between the combinatorial problem, $V(\tilde{m})$, and the relaxed problem, $V\left(M \lambda^{*}\right)$, gets small already for

\footnotetext{
${ }^{7} \mathrm{~A}$ covariance value of 1 corresponds to $\Phi_{v}\left(\omega_{k}\right) / \Phi_{r}\left(\omega_{k}\right) \approx 58 \cdot \omega_{k}^{2}$, which means a really poor SNR. In the real application, the SNR is large which will give a much better parameter accuracy.
}

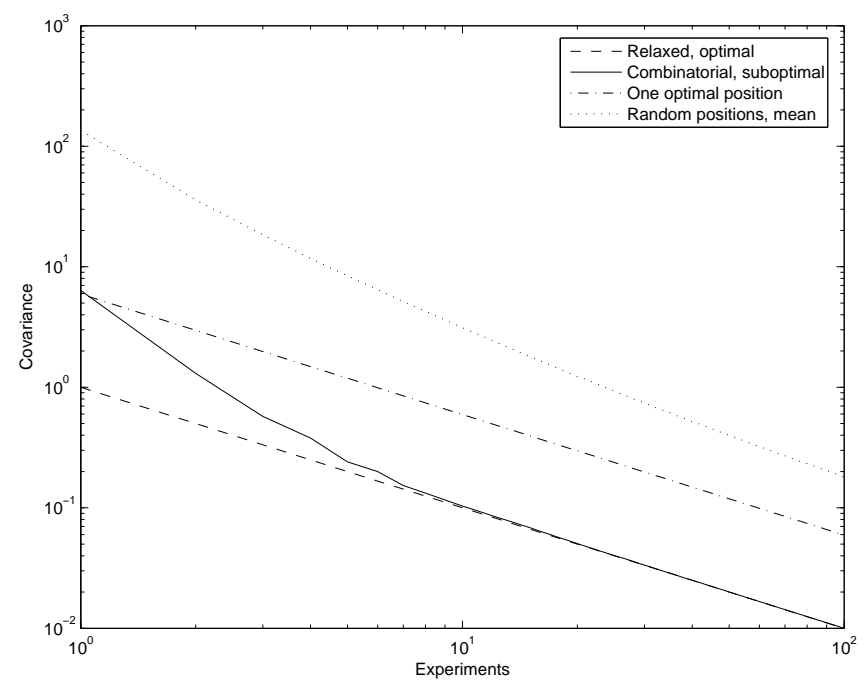

Fig. 4. Covariance, $(\operatorname{det} H)^{-1 / d}$, as a function of the number of experiments $M$.

quite small $M$ values $(M \approx 10)$. The accuracy obtained by performing $M=10$ experiments distributed over the optimal positions (then actually $\leq 10$ positions) corresponds to almost 60 experiments in the optimal single position. Or the other way, 3 experiments in different positions give the same accuracy as 10 experiments in the optimal single position.

In addition, using multiple positions will probably make the experiment design more robust to uncertainties in $\theta_{0}$, but that analysis is left for future work. To robustify the experiment design, one could also add the convex constraint that no more than a certain fraction of the $M$ experiments is concentrated in less than a given fraction of the $Q$ possible experiments, see [11, Section 2.4] for details ${ }^{8}$.

If the user has no knowledge about the system, multiple random positions might be a good alternative, compared to using one random position, which is indicated by the dotted curve in Figure 4. Using 1 random position for $M=100$ experiments will in average give 10 times worse accuracy compared to using 100 random positions. This might not be so surprising, but it is interesting to notice that with $M=$ 100 , the optimal single position is only 3 times better than using 100 random positions.

\section{CONCLUSIONS}

Experiment design involving selection of optimal experiment positions has been treated. From the derived Fisher information matrix, a convex optimization problem is posed. By considering the dual problem, the experiment design is efficiently solved with linear complexity in the number of candidate positions, compared to cubic complexity for the primal problem. In the numerical illustration, the 15 optimal positions reduces the parameter uncertainty by a

\footnotetext{
${ }^{8}$ Unfortunately, the structure in the design problem that was exploited to derive an efficient dual formulation will typically be lost when adding additional complicated constraints.
} 


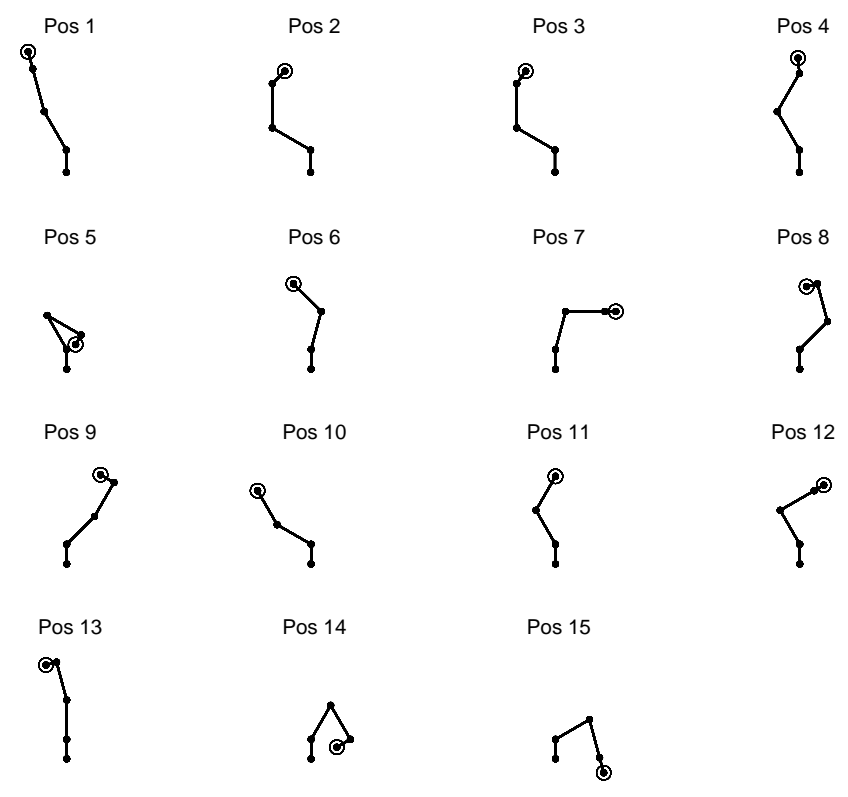

Fig. 5. Illustration of the optimal positions from Table I, here viewed from the side. The payload is marked by a circle.

factor of 6, compared to using the optimal single position in all experiments.

\section{ACKNOWLEDGMENTS}

This work has been supported by ABB Robotics and Vinnova's Center of Excellence ISIS at Linköpings universitet. The authors also want to thank Martin Enqvist for inspiring discussions.

\section{REFERENCES}

[1] V. V. Fedorov, Theory of optimal experiments. Academic Press, New York, 1972.
[2] G. C. Goodwin and R. L. Payne, Dynamic System Identification: Experiment Design and Data Analysis. Academic Press, New York, 1977.

[3] M. Gevers, "Identification for control: From the early achievements to the revival of experiment design," European Journal of Control, vol. 11, no. 4-5, pp. 335-352, 2005.

[4] L. Ljung, System Identification: Theory for the User, 2nd ed. Upper Saddle River, New Jersey, USA: Prentice Hall, 1999.

[5] G. C. Goodwin, J. S. Rojas, and J. S. Welsh, Forever Ljung in System Identification. Studentlitteratur, Lund, 2006, ch. Good, Bad and Optimal Experiments for Identification.

[6] H. Jansson and H. Hjalmarsson, "Input design via LMIs admitting frequency-wise model specifications in confidence regions," IEEE Transactions on Automatic Control, vol. 50, no. 10, pp. 1534-1549, Oct 2005.

[7] J. Öhr, S. Moberg, E. Wernholt, S. Hanssen, J. Pettersson, S. Persson, and S. Sander-Tavallaey, "Identification of flexibility parameters of 6-axis industrial manipulator models," in Proc. 2006 International Conference on Noise and Vibration Engineering (ISMA 2006), Leuven, Belgium, Sep 2006.

[8] R. Pintelon and J. Schoukens, System identification: a frequency domain approach. New York: IEEE Press, 2001.

[9] J. Löfberg, "YALMIP : A toolbox for modeling and optimization in MATLAB," in Proceedings of the CACSD Conference, Taipei, Taiwan, 2004. [Online]. Available: http://control.ee.ethz.ch/ joloef/yalmip.php

[10] S. Boyd and L. Vandenberghe, Convex Optimization. Cambridge University Press, 2004.

[11] L. Vandenberghe, S. Boyd, and S.-P. Wu, "Determinant maximization with linear matrix inequality constraints," SIAM Journal on Matrix Analysis and Applications, vol. 19, no. 2, pp. 499-533, 1998.

[12] A. Ben-Tal and A. Nemirovski, Lectures on Modern Convex Optimization: Analysis, Algorithms, and Engineering Applications, ser. MPSSIAM series on Optimization. Philadelphia, Pennsylvania: SIAM, 2001.

[13] K. C. Toh, R. H. Tütüncü, and M. J. Todd, "On the implementation and usage of SDPT3 - a MATLAB software package for semidefinitequadratic-linear programming, version 4.0," Jul 2006, available from http://www.math.nus.edu.sg/ mattohkc/guide4-0-draft.pdf.

[14] K. Aström and B. Wittenmark, Computer Controlled Systems - Theory and Design. Prentice-Hall, Englewood Cliffs, NJ, 1984.

[15] E. Wernholt and S. Gunnarsson, "Analysis of methods for multivariable frequency response function estimation in closed loop," Department of Electrical Engineering, Linköping University, SE-581 83 Linköping, Sweden, Tech. Rep. LiTH-ISY-R-2775, Mar 2007, submitted to CDC'07. 



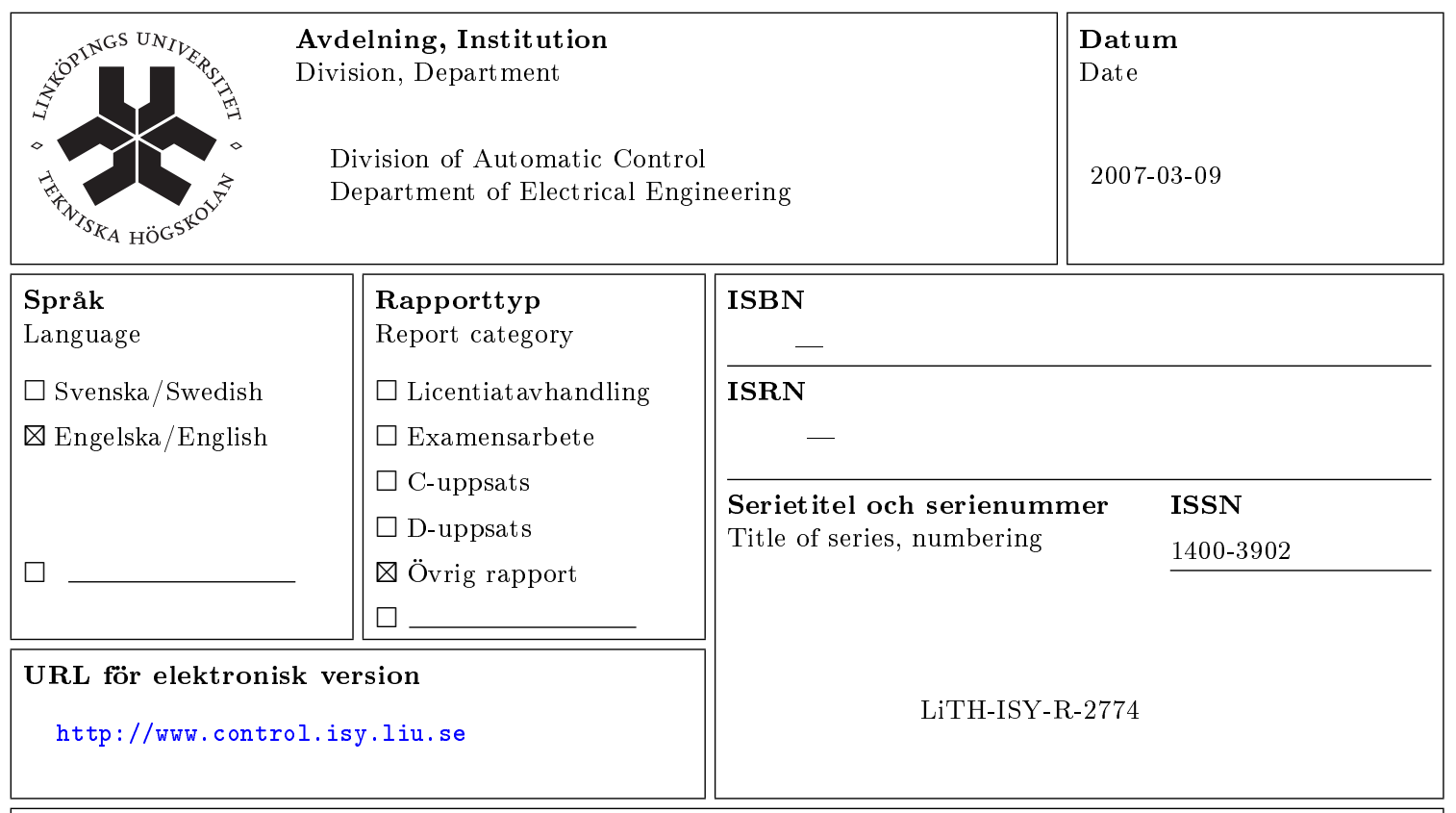

Titel Experiment Design for Identification of Nonlinear Gray-box Models with Application to Title Industrial Robots

Författare Erik Wernholt, Johan Löfberg

Author

\section{Sammanfattning}

Abstract

Experiment design involving selection of optimal experiment positions for nonlinear graybox models is studied. From the derived Fisher information matrix, a convex optimization problem is posed. By considering the dual problem, the experiment design is efficiently solved with linear complexity in the number of candidate positions, compared to cubic complexity for the primal problem. In the numerical illustration, using an industrial robot, the parameter covariance is reduced by a factor of six by using the 15 optimal positions compared to using the optimal single position in all experiments. 